\title{
Cétoines, magie noire et divination au Mali
}

\section{Henri-Pierre Aberlenc}

Abstract

Cetonid beetles, black magic and divination in Mali

In Mali, the Diplognatha gagates cetonid beetle is considered as the messenger of an ill-intentioned sorcerer. The Rhabdotis sobrina, Pachnoda cordata and P. marginata aurantia cetonid beetles predict the coming of someone.

\section{Résumé}

Au Mali, la cétoine Diplognatha gagates est perçue comme la messagère d'un sorcier malintentionné. Les cétoines Rhabdotis sobrina, Pachnoda cordata et P. marginata aurantia annoncent quant à elles la venue d'un visiteur.

\section{Citer ce document / Cite this document :}

Aberlenc Henri-Pierre. Cétoines, magie noire et divination au Mali. In: Bulletin mensuel de la Société linnéenne de Lyon, $66^{\mathrm{e}}$ année, $\mathrm{n}^{\circ} 10$, décembre 1997. pp. 277-278;

doi : https://doi.org/10.3406/linly.1997.11420

https://www.persee.fr/doc/linly_0366-1326_1997_num_66_10_11420

Fichier pdf généré le 28/03/2018 
Bull. mens. Soc. linn. Lyon, 1997, 66 (10) : 277-278.

\title{
Cétoines, magie noire et divination au Mali
}

\section{Henri-Pierre Aberlenc}

CIRAD, Laboratoire de Faunistique et de Taxonomie, BP 5035, 34032 Montpellier Cedex 1.

\begin{abstract}
Résumé. - Au Mali, la cétoine Diplognatha gagates est perçue comme la messagère d'un sorcier malintentionné. Les cétoines Rhabdotis sobrina, Pachnoda cordata et $P$. marginata aurantia annoncent quant à elles la venue d'un visiteur.

Mots-clés. - Ethno-entomologie, Mali, magie noire, divination, cétoines.
\end{abstract}

\section{Cetonid beetles, black magic and divination in Mali}

Summary. - In Mali, the Diplognatha gagates cetonid beetle is considered as the messenger of an ill-intentioned sorcerer. The Rhabdotis sobrina, Pachnoda cordata and $P$. marginata aurantia cetonid beetles predict the coming of someone.

Key words. - Ethno-entomology, Mali, black magic, divination, cetonid beetles.

En Afrique, la croyance en des forces occultes omniprésentes est très répandue. La rencontre ou la capture de certaines espèces d'arthropodes (arachnides, crustacés, myriapodes ou insectes) peut, en fonction de la personne concernée, du lieu et du moment, être hautement signifiante : un tel événement peut avoir valeur d'augure, de porte-bonheur, de message de l'au-delà envoyé par les Ancêtres, provoquer un mal que seul un rituel pourra écarter (FERRY, 1980 ; SEIGNOBOS et al., 1996), etc. Certains rituels de divination, pratiqués par des devins (PARÉ, 1956) ou par les intéressés euxmêmes (DENIS, 1964) nécessitent l'emploi d'une espèce précise.

Le simple recensement de ces savoirs traditionnels, le plus souvent propres à une région ou à une ethnie, aurait le volume d'une encyclopédie. Beaucoup serait à faire en ce domaine, car les lacunes de nos connaissances sont considérables. Diverses raisons peuvent les expliquer : l'ampleur du sujet, le secret initiatique qui interdit de révéler certaines choses, et, il faut le dire, le peu d'intérêt des ethnologues et des géographes pour ce qui touche au monde des insectes. De plus, on peut constater que nombre de jeunes Africains urbanisés ou en voie de l'être n'écoutent plus les Anciens et se désintéressent de ces savoirs traditionnels (SEIgNobos et al., 1996).

Lors d'un séjour au CIRAD à Montpellier en 1994, notre Collègue Boubakary CISsÉ nous apprit l'existence de certaines croyances traditionnelles de son pays. Si ces conceptions sont générales au Mali, elles sont combattues par les grandes religions monothéistes, l'Islam et le Christianisme. Mais,

Accepté pour publication le 15 mai 1997.

Bull. mens. Soc. linn. Lyon, 1997, 66 (10). 
comme souvent en Afrique, le monothéisme le plus orthodoxe peut coexister, dans la vie quotidienne, avec des conceptions et des pratiques traditionnelles que l'on qualifie, faute d'un meilleur terme, d' « animistes».

\section{MAGIE NOIRE}

La notion de magie noire ne se rapporte évidemment pas à la couleur de la peau de celui qui s'y adonne mais aux finalités et aux moyens de telles pratiques occultes.

Il peut arriver que l'on soit heurté en plein vol par la cétoine Diplognatha gagates (figure 1), qui tombe alors à terre. On peut également être cogné sans voir l'insecte. Dans les deux cas, on sait alors que ce dernier est envoyé par un sorcier qui a jeté un mauvais sort. Un mal incurable et mortel se déclarera. Si on connaît le sorcier, on peut lui demander de revenir sur sa malédiction et de la retirer. Sinon il est nécessaire de recourir à un exorciste.

\section{DIVINATION}

Certaines cétoines jouent le rôle d'augures. Quand on rencontre chez soi ou dans sa cour, soit la cétoine Rhabdotis sobrina (figure 2), soit Pachnoda cordata (figure 3 ), soit encore $P$. marginata aurantia (figure 4 ), on est certain de la venue proche d'un visiteur.

Remerciements. - Nous remercions nos Collègues Boubakary CIssÉ pour ses informations, Roger Roy et Christine Devaud pour de la documentation, Stéphane BIROT pour les photos, sans oublier Didier BARTHÉlÉmY pour son aide technique.

\section{RÉFÉRENCES BIBLIOGRAPHIQUES}

DENIS J., 1964. - L'Araignée rend la justice... L'Entomologiste, 20 (1-2) : 15-17, 1 fig.

FERRY M.-P., 1980. - Principaux invertébrés connus des populations du département de Kédougou (Sénégal). Bulletin de l'I.F.A.N., Série B, 42 (3) : 502-523.

PARÉ I., 1956. - L'araignée divinatrice. Etudes camerounaises, 53-54 : 61-83.

Seignobos C., Deguine J.-P. et Aberlenc H.-P., 1996. - Les Mofu et leurs insectes. Journal d'Agriculture Traditionnelle et de Botanique Appliquée, 38 (2) : 125-187, 36 fig.

Légendes des illustrations (photographies de S. BIRor) :

Fig. 1 - Diplognatha gagates (Forster).

Fig. 2 - Rhabdotis sobrina (Gory et Percheron).

Fig. 3 - Pachnoda cordata Drury.

Fig. 4 - Pachnoda marginata aurantia Herbst. 

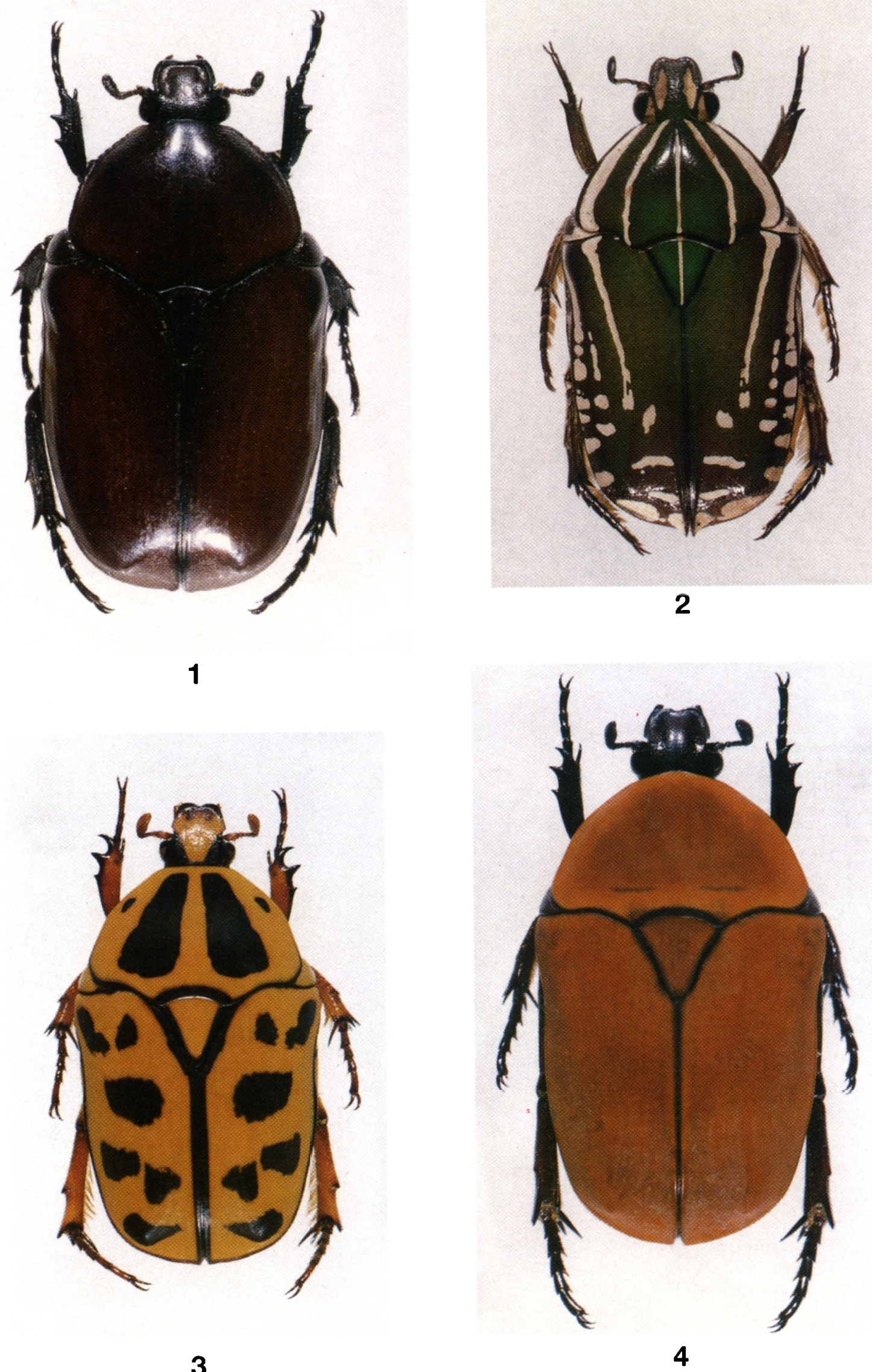

Bull. mens. Soc. linn. Lyon, 1997, 66 (10). 